\title{
Nota al artículo "El sistema de las Creative commons"
}

\section{Por Ignasi Labastida i Juan}

DESPUÉS DE LEER EL ARTÍCULO "El sistema de las Creative Commons" de Marco Marandola ${ }^{1}$, me gustaría presentar de manera más completa el proyecto de las licencias de Creative commons. Actualmente las palabras copyleft, copyright, open access, creative commons, procomún, se utilizan mucho pero a veces se mezclan conceptos y se informa de manera errónea. Agradezco a los editores la posibilidad de escribir esta nota que personalmente considero de rectificación.

Creative commons nació en el año 2001 y el 16 de diciembre de 2002 se hizo público el proyecto más conocido hasta ahora, el de las licencias. La intención era facilitar unos textos legales para que los autores pudieran ceder algunos derechos sobre sus obras y se pudieran reservar otros. Creative commons no está contra los derechos de autor, sino todo lo contrario, de hecho su objetivo es promover la cultura ayudando a los creadores a ejercer sus derechos.

Hay que dejar bien claro que el uso de las licencias es completamente gratuito y que no hace falta registrar la obra en ningún repositorio específico.

Para aclarar algunos aspectos centraré la explicación en las licencias estándar, que son aquellas a las que se accede desde el apartado "Publish" de la web de Creative commons $^{2}$. El creador sólo tiene que responder a dos preguntas: si quiere permitir un uso comercial de la obra y si permite la generación de otras derivadas de ella. Además puede escoger la jurisdicción más adecuada, como veremos más adelante, y rellenar un formulario de datos sobre el documento. La negativa a un uso comercial no impide que el autor comercialice su obra de la manera que crea más conveniente; aquí lo que está cediendo - o no- es el derecho a que otra persona haga este uso libremente. Si el autor permite la generación de obras derivadas puede imponer que éstas se encuentren sujetas a una licencia del mismo tipo. Este último punto no entra en conflicto con la jurisdicción española, ya que no se trata de una cesión de derechos mo- 
rales, sino del ejercicio del artículo 21.2 de la Ley de Propiedad Intelectual:

"Los derechos de propiedad intelectual de la obra resultado de la transformación corresponderán al autor de esta última, sin perjuicio del derecho del autor de la obra preexistente de autorizar, durante todo el plazo de protección de sus derechos sobre ésta, la explotación de esos resultados en cualquier forma y en especial mediante su reproducción, distribución, comunicación pública o nueva transformación"”.

De la combinación de respuestas a estas dos preguntas se obtienen las seis licencias estándar actuales, de las cuales hablaré en detalle más adelante.

La primera característica de las licencias es la triple lectura: el resumen (commons deed -escritura común-), el texto legal (legalcode) y el código o metadatos (digital-(-ode $)^{4}$. En el resumen se puede encontrar una explicación breve y concisa de qué y cómo se puede hacer con la obra sujeta a la licencia. El texto legal es la licencia propiamente dicha y finalmente el código sirve para incrustarlo en cualquier página web donde se ofrezca la obra, siempre que esté online. Esta última lectura de las licencias está pensada para materiales digitales pero el uso de las licencias no excluye otro tipo de soportes más tradicionales como el papel, tal cual se ha visto en muchos ejemplos ${ }^{5}$.

Así pues, las licencias están pensadas para contenidos y no para soportes, es decir que una obra en papel, pdf o html puede utilizar la misma licencia.

Respecto a su utilización para software, en ningún apartado de las licencias se especifica condición alguna sobre el código fuente de la obra. Por esta razón, desde la misma web de Creative commons se aconseja utilizar otros tipos de li- cencia creados específicamente para ello, como por ejemplo las de la Free Software Foundation (FSF) o la Open Source Initiative ${ }^{6}$.

De hecho, desde la sección de las licencias se puede acceder a unos resúmenes de las licencias de la $F S F$, con el mismo aspecto que los "deed" de Creative commons?. No obstante el enlace al texto legal es a la licencia oficial de la FSF.

\section{Las licencias estándar}

El texto de las licencias es una cesión de algunos de los derechos de autor. Sólo se ceden algunos de los derechos de explotación ya que en ciertas jurisdicciones, como la española, existen los derechos morales que son inalienables y no se pueden ceder.

Las seis existentes permiten la copia, distribución y comunicación de las obras siempre que se cumplan las condiciones establecidas por el autor o licenciador. Actualmente todas ellas requieren el reconocimiento del autor original de la obra y de aquellas partes que el autor o licenciador designe. Las licencias son de ámbito mundial, sin derecho de remuneración, no exclusivas e indefinidas. La cesión de derechos se extiende a la vigencia de los derechos de propiedad intelectual establecida por la ley y es irrevocable, aunque el licenciador se reserva el derecho a divulgar o publicar la obra en condiciones distintas o incluso a retirarla. En este caso la licencia continuará vigente para aquellos que hubieran recibido la obra con anterioridad.

Otra de las condiciones que establecen todas las licencias es dejar bien claro los términos de la licencia de esta obra al copiarla o distribuirla. Las restricciones que se aplican en la copia, distribución y comunicación pública vienen determinadas por el tipo de licencia escogida $^{8}$ :
-Reconocimiento (by): se permite su uso comercial, así como el de las posibles obras derivadas, cuya generación y distribución está permitida sin ninguna restricción.

-Reconocimiento-NoComercial $(b y-n c)$ : es posible la generación de obras derivadas siempre que no se haga un uso comercial. Tampoco puede utilizarse la obra original con fines comerciales.

-Reconocimiento-NoComercial-CompartirIgual (by-nc-sa): no se permite un uso comercial de la obra original ni de las posibles obras derivadas, cuya distribución debe hacerse mediante una licencia igual que la sujeta a la obra original $(s a=$ share alike $)$.

-Reconocimiento-NoComercial-SinObraDerivada (by-nc-nd): no se consiente un uso comercial de la obra original ni la generación de otras derivadas.

-Reconocimiento-CompartirIgual (by-sa): se admite el uso comercial de la obra y de las derivadas, cuya distribución debe hacerse mediante una licencia igual que la sujeta a la obra original.

-Reconocimiento-SinObraDerivada (by-nd): se permite el uso comercial de la obra pero no la generación de derivadas.

Tal como se indica en los resúmenes de las seis licencias, alguna de estas restricciones puede ser revocada si se obtiene la autorización expresa del titular de los derechos. Es decir, si alguien quiere hacer un uso comercial de una obra, llegando a un acuerdo con el licenciador se podrá obtener este derecho de explotación específico.

Además de estas licencias estándar, desde la página web de Creative commons se puede acceder a otros tipos de licencias":

-Public domain. Es una cesión al dominio público basada en la jurisdicción de EUA como se indica 


\section{Años de experiencia}

Esto es lo que EBSCO ofrece.

Nuestro personal gestiona sus suscripciones a revistas electrónicas individuales o incluidas en paquetes de revistas, suscripciones en papel y bases de datoś.

La Lista A-Z (A-to-Z) agrupa todos: sus recursos electrónicos en una misma lista, para que sus usuarios localicen de manera rápida los títulos disponibles y accedan fácilmente a los contenidos. Además, la lista A-to-Z alimenta ál servidor de enlaces LinkSource ${ }^{m}$, que utilizando la norma OpenURL enlaza todos sus recursos de forma compacta e inteligente.

Experiencia, servicio, contenido, soluciones. Hablemos hoy de sus necesidades.

CUSTOMERFOCUSEDCONTENTDRIVEN:

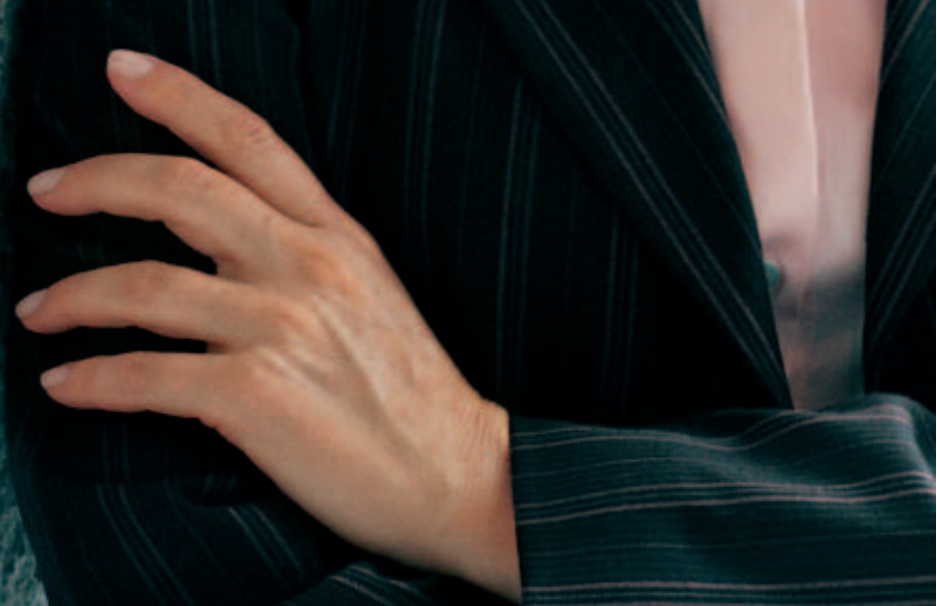
www.ebsco.com
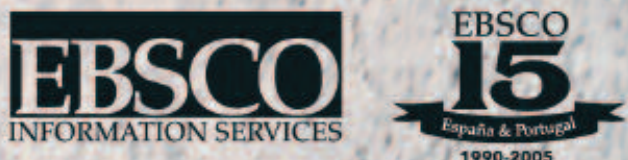
al principio. Con dicha jurisdicción es posible renunciar a los derechos sobre una obra y que ésta pase al dominio público antes de que se acabe la protección.

http://creativecommons.org/license s/publicdomain/

—Developing nations. Licencia pensada para permitir usos sobre la obra sólo en los países en desarrollo.

http://creativecommons.org/license s/devnations/2.0/

-Sampling. Se trata de tres licencias diferentes pensadas para todos aquellos materiales susceptibles de ser mezclados u obtener muestras útiles de ellos: música, vídeo, imagen, etc.

http://creativecommons.org/license /sampling

-Founder's copyright. Está basada en la primera licencia sobre copyright de los EUA, de 1790, mediante la cual la extensión de los derechos sobre una obra era de 14 años prorrogable a otros 14 .

http://creativecommons.org/project s/founderscopyright/

-Share music. Es equivalente para música a Reconocimiento-No Comercial-SinObraDerivada.

http://creativecommons.org/license s/by-nc-nd/2.0/legalcode

\section{Internacionalización de las licencias}

Cuando se inició el proyecto los textos que se ofrecían desde la web estaban basados en la legislación americana sobre copyright. Desde un principio se pensó en la manera de llevar a cabo una adaptación a las diferentes jurisdicciones. Así nació a principios de 2003 el proyecto iCommons $^{10}$ con la participación de Brasil, Finlandia y Japón. El proyecto Creative Commons España se gestó en la primavera de 2003 a partir de los primeros contactos entre Creative commons y la Universitat de Barcelona. La intención de la $U B$ era entonces disponer de una licencia equivalente a la del Opencourseware del $M I T^{11}$ para los materiales docentes generados por el propio profesorado. Además de obtener el permiso para utilizar unas licencias específicas, se nos propuso liderar el proyecto de adaptación de las licencias y de esta manera la $U B$ se convirtió en la institución afiliada a Creative commons en España. El primer borrador de una de ellas se expuso a debate público a partir de febrero de 2004 a través de una lista de discusión de acceso libre. Todo el proceso terminó el 1 de octubre de 2004, fecha a partir de la cual cualquier autor puede escoger la jurisdicción española en la página de Creative commons y disponer de unos textos adaptados a la ley de propiedad intelectual vigente en España ${ }^{12}$.

Así pues iCommons no sólo consiste en traducir los textos sino que es necesario adaptarlos a las diferentes jurisdicciones intentando mantenerlos lo más similares posible. Además, la internacionalización del proyecto permite que las diferentes actividades de Creative commons se extiendan a otros territorios y que se construyan comunidades locales con proyectos pro$\operatorname{pios}^{13}$.

\section{Conclusión}

El sistema de licencias Creative commons, de la misma manera que la ley, no garantiza la protección sobre las obras. Siempre habrá quien no haga un buen uso por desconocimiento o con toda la intención. De todas formas lo que se pretende es que el acceso a las creaciones sea más sencillo y que el autor pueda ceder a priori unos derechos que por ley son suyos. Los límites de utilización de las obras los debe marcar el autor.

Este proyecto de las licencias no está finalizado, se encuentra siempre abierto para recoger las inquietudes y necesidades de los creadores. De esta manera se pueden ir versionando los textos e incluso crear nuevas licencias para fines más específicos. Pero además hay otros proyectos de Creative commons como CCMixter, dedicado a las obras musicales, o Science commons, centrado en el ámbito de la ciencia, que poco a poco van adquiriendo importancia y que merecerían un artículo entero para ser explicados correctamente.

\section{Notas}

1. Marandola, Marco. "El sistema de las Creative commons". En: El profesional de la información, 2005, julio-agosto, v. 14, n. 4, pp. 285 289.

http://elprofesionaldelainformacion.metapress.c om/openurl.asp? genre $=$ article \&issn $=1386$ $6710 \&$ volume $=14 \&$ issue $=4 \&$ spage $=285$

2. http://creativecommons.org/license/

3. Texto refundido de la Ley de propiedad intelectual, 12 abril de 1996.

http://www.mcu.es/propint/files/LeyProp_Intelec tual_mod172.pdf

4. Un ejemplo de esta triple lectura para la licencia Reconocimiento 2.1 España.

Common deed:

http://creativecommons.org/licenses/by/2.1/es/de ed.es

Texto legal:

http://creativecommons.org/licenses/by/2.1/es/le galcode.es

Código:

http://creativecommons.org/license/work-htmlpopup?lang $=e s \& j u r i s d i c t i o n=e s \& l i c e n s e \_c o d e$ $=b y$

5. Un par de ejemplos locales:

- Software libre, Jordi Mas.

http://www.infonomia.com/textos/software.asp

- La taberna errante, G. K. Chesterton.

Papel:

http://acuareladiscos.com/libros/catalogo.htm $\mathrm{Pdf} / \mathrm{ps}$ :

http://sindominio.net/biblioweb/literatura/flyingi nn-es/

—Virtuosismo y revolución, Paolo Virno:

http://www.sindominio.net/traficantes/virtuosism o.htm

6. Página de FAQ de Creative commons: http://creativecommons.org/faq\#faq_entry_3646

7. Por ejemplo, el resumen de la licencia GPL: http://creativecommons.org/license/cc-gpl

8. Las licencias adaptadas a la legislación española están disponibles en la web de Creative commons:

http://creativecommons.org/licenses/xxx/2.1/es/ donde "xxx" tiene que ser sustituido por by, by $n c, b y-n c-n d, b y-n c-s a, b y-n d, b y-s a$ según corresponda.

9. Las otras licencias pueden encontrarse en: Public domain: http://creativecommons.org/licenses/publicdoma in/

Developing nations: 
http://creativecommons.org/licenses/devnations/ 2.0/

Sampling:

http://creativecommons.org/license/sampling

Founder's copyright:

http://creativecommons.org/projects/foundersco pyright/

Share music:

http://creativecommons.org/license/music
10. Proyecto iCommons:

http://creativecommons.org/worldwide/

11. OpenCourseWare:

http://ocw.mit.edu

12. Cueva, Javier de la. “¿Por qué las licencias Creative commons son legales en España?"

Texto leído en la conferencia de los 100 días de

las licencias Creative commons España, Madrid 24 enero de 2005. http://www.derecho-internet.org/node/272

13. Actualmente hay dos sitios web en el estado español:

http://es.creativecommons.org

http://cat.creativecommons.org

Ignasi Labastida i Juan, responsable de Creative Commons España.

ilabastida@ub.edu 